\title{
Power enhancement based link quality for wireless mesh network
}

\author{
Mohammad Khalaf Rahim Al-juaifari ${ }^{1}$, Hussain Mumtaiz Alshamy ${ }^{2}$, Noor Hassan Abed Khammas ${ }^{3}$ \\ ${ }^{1}$ ITRDC, University of Kufa, Iraq \\ ${ }^{2,3}$ Engineering Science, Electronic and Communication Department, Kufa University, Iraq
}

\begin{tabular}{|c|c|}
\hline Article Info & ABSTRACT \\
\hline Article history: & $\begin{array}{l}\text { Energy consumption of wireless network communication is still a big issue } \\
\text { and a lot of research papers have proposed many solutions to increase node }\end{array}$ \\
\hline Received Feb 14, 2020 & life time. The WMN architecture is made up of a fixed and mobile \\
\hline Revised Sep 3, 2020 & component, whereas the wireless mesh networks (WMNs) are multi-hop \\
\hline Accepted Oct 1, 2020 & $\begin{array}{l}\text { wireless networks with instant deployment, self-healing, self-organization } \\
\text { and self-configuration features. The reduction in the distance by a factor of }\end{array}$ \\
\hline Keywords: & $\begin{array}{l}\text { two can result in at least four times more powerful signals at the receiver. } \\
\text { This paper presents suggestions that the links are more reliable without the }\end{array}$ \\
\hline Energy consumption & increase in power of the transmitter in individual nodes. As a result, the \\
\hline Link quality & $\begin{array}{l}\text { present simulations networks are nine mobile nodes for considering coverage } \\
\text { issues of the service area. The analytic results show that the link power node }\end{array}$ \\
\hline Power control & for direct communication between two nodes with long distance consuming \\
\hline WMN: $802.11 \mathrm{~s}$ & more power than it is cleared. The improvement in the network performance \\
\hline WMNs & $\begin{array}{l}\text { for maintaining is available and this solution can be used to implement mobility in } \\
\text { such case with low power region for the wireless mesh networks. }\end{array}$ \\
\hline
\end{tabular}

This is an open access article under the CC BY-SA license.

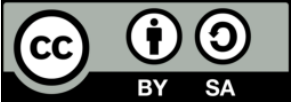

Corresponding Author:

Mohammad Khalaf Rahim Al-juaifari

ITRDC, University of Kufa

Kufa, Iraq

Email: Mohammad.aljuaifari@uokufa.edu.iq

\section{INTRODUCTION}

WMNs offer multiple redundant communications paths throughout the network and when a link fails, the network automatically routes messages through alternate paths even in adverse conditions. The devices in WMNs cooperate with each other for transmitting packets through the network. WMNs are consider a self-configuring and self-healing networks and the dramatic increase in link quality required shortening the distance between the nodes. There are different types of energy consumption in the network such as an active, idle, off, also the minimum hops; error rate, delay, maximum data rates and route stability are included with power consumption. The WMNs architecture that described in Figure 1 is mainly depends on the node and divided into three types. The first one is infrastructure/backbone WMNs, which is the collection of mesh routers of the gathering packet traffic from mobile or static clients then forward it to the main network or ISP. The second is the client WMNs nodes that do routing, configuration, and also the user applications, while the third one is mesh user.

The problems in WMNs such as interference, mobility and bandwidth are cause in link communication failure. Then there are many uses offer some features such as a self-organized, self-healing and reconfigurable to be used in solving such problem in WMNs. A proportional power with low cost is provided while maintaining flexible deployment with decent coverage and robustness to make WMNs suitable for incident situations [1]. 


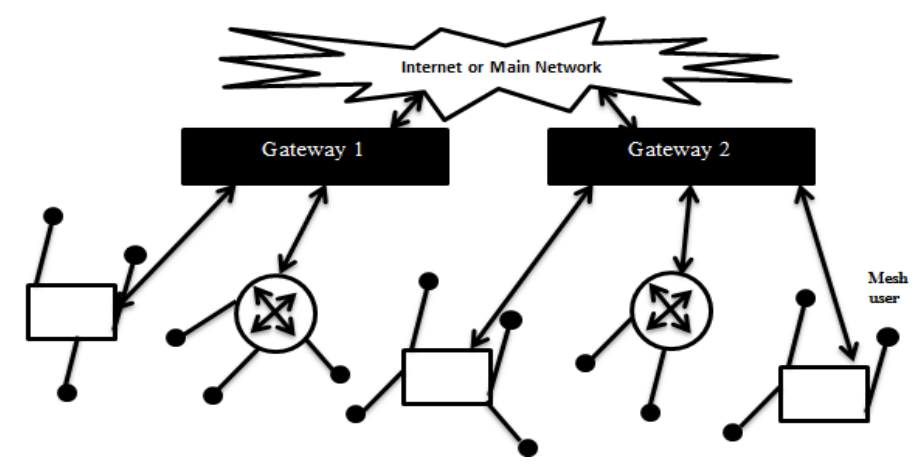

Figure 1. Hybrid wireless mesh network architecture (with ability to both of internet service providers (ISPs) and wireless users' requests

The main contribution of this work is by choosing the next node as a bridge rather than communication directly. The energy methods in WMNs for the physical layers can be divided into: a dynamic voltage control designed to allocate low power for low load of the processor and traffic requirements. Then the node with level power scheduling the control power for transmission power is varied at each node by control topology and by choosing a minimum subset.

A smaller number of hops (neighbors) are used to complete packet from transmitting to receiving, while the option for the sleep schedules of the buffering nodes which are in sleep mode is by buffering and managing delay packets. The option of reducing power consumption and keep connection alive as long as possible, especially in outdoor places with less power supplies is by selection of optimal node for the transmitter with power levels to keep network a live as long as possible. In the same time the transmission energy is saved in such case for long distance which is required for high speed packet to transfer along with environment.

Recently, the power aware solutions for WMNs have become an important issue especially in low power regions. Most MAC protocols used a proposed active/sleep energy saving in WMNs with network allocation vector (NAV) [2]. An automatic power saves delivery (APSD) [3] happen when the link is busy as the block stations is busy from transmitting. Another case is the link is called an active/sleep schedules when the ability to improve flexibility and power saving. The power saver of multi-poll (PSMP) [4] is an enhancement, while for the downlink and uplink transmissions are used to minimize the overhead of active/sleep schedules of the U-APSD and S-APSD protocols by scheduled times. A sleep optimal fair-attention scheduler (SOFA) [5] combines of both Active/sleep and round robin scheduling. It is happened by make responsiveness requests for clients to sleep rather than scheduling policies. The energy aware routing (EAR) [6] route packet traffic which calculate the energy consumption for component.

The throughput-aware routing (ETR) [7] chooses the routing and depends only the shortest path. The nodes become an active by switching off as much nodes as much as possible. The green framework [8] is routes with Link Scheduling, and green routing framework with link scheduling had been proposed for WMNs. While CBRP [9] is routes with reduce overflowing traffic and this protocol route the discovery for WMNs by clustering to reduce overflow. The function of the control and localized distribution topology for hybrid WMN of heterogeneous to choose the best power path for the neighbor node was presented in [10], in [11] the signal strength calculations had been calculated, and the guarantee network connectivity also the time of the transmission energy is saved. While determine the quality in any direction of the link in [12]. In [13] divide one frame to small slots for sensor nodes in WMSN then forward data packets to mesh router which computes the slot allocation table for collecting information from neighbor, also in [14] a new framework had been proposed to enhance power saving by present both planning and energy management solution in WMN at same time. While in [15] save energy by proposing a routing protocol that reduces the packet fallout. Dynamic energy management presented in [16] by time varying dynamic selection of base stations along with routing of WMNs. In [17] almost all the protocols and many more have been revised, where the data and the network layers cannot save power only in physical layer in which devices is exploited. Also calculated whether the traffic through ACMR and GMR of the link can go to be active, otherwise it should be in sleep. In [18] introduced an online flow-based routing traffic due to energy efficient communication in WMNs. A bluetooth low energy or bluetooth smart had been merged technologies and the methods for wireless network in [19], also in [20] presented an industrial solution for low power consumption component in WMNs. Authors in [21] has an infinite set of physical links to use a physical interference mode for single hop and multi-hops to reach an optimal power consumption by discrete set of data rates with continuous power control. 
The result in [22] for the simulations shows that whenever one link fails to produce full robustness and when the link failure in the network a fault tolerance will be tolerate from $29.71 \%-45.61 \%$. In [23] make a node works out of power with MP-CARA falls with less than a desired threshold. The dynamic STA in [24] with save substantial energy has an aggregate battery by random mobile points in a WMN. Authors in [25] proposed algorithms to minimize network overheads, maintaining a high delivery ratio, and low latency to increase network performance.

\section{RESEARCH METHOD}

This work is designed for controlling the paths based on power whenever a node A is sending a packet along the path to neighbor node B. In Figure 2 when a packet Hello sends the same power level the routes are selected based on the power of particular node. The Packet route through the distance between the nodes along the path (A, D, G, H and I) rather that path (A, C, F and I) with the same level of power through all nodes. The distance from route $\mathrm{F}$ mobile node to I destination, the power consumption is greater than power consumption via route $\mathrm{G}, \mathrm{H}$, and I considering same distance. The results of this can be seen on the simulation which is part of this paper where a constant number of metrics for the power consumption in Figure 3 shows the proposed work.

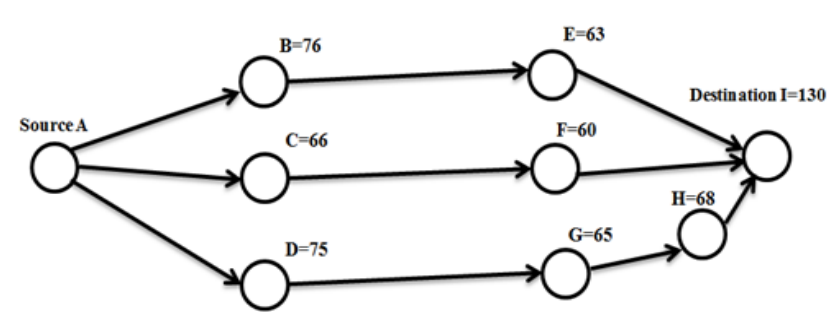

Figure 2. Coverage of distance-based sample system

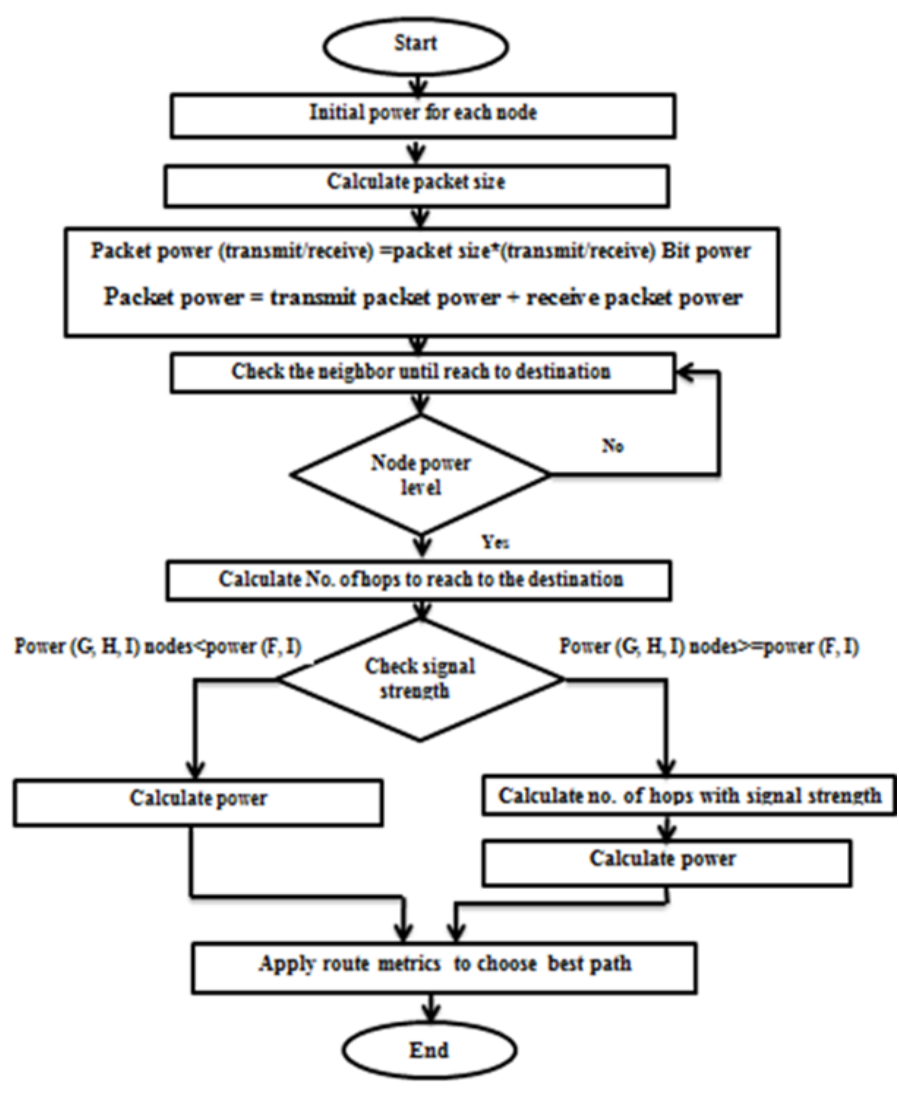

Figure 3. Flowchart of proposed method 
The initial power of network node with the power consumption of the receiver/transmitter and the movement of the constant power, with the parameters of the initial power and remaining power $=\Sigma$ Initial power-node power consumed, battery-life status, type of data transfer, and node type priority. Both the routing of the mesh router and mesh clients with physical link quality help in selecting of the stability, high bandwidth and low error rate routes. The fading signal can affect the strength of the signal and a link about to fail preemptive in the route requests. The energy consumption at physical layer is proportional to the traffic load and it is developed to be an active link based on node selected, also the control of continues power to be on the maximum power and less than power of one hop.

\section{RESULTS AND DISCUSSIONS}

The implementation of this work in real time life and a real sample has been taken which belongs to existing buildings at Kufa University. The result of this experimental depends on some parameters such as a distance between the nodes, and signals between the buildings of the Kufa University. The distance from node A to I is shown in Figures 1-3 respectively and the formula (1) is obtained by using the distance in parameters Table 1 along with Table 2 and the power in Table 3.

$$
\text { Pt./ D. (node } i, j) \text { Ce }>=S t \text {. }
$$

where Pt. represents the transmit power, D. represents the distance, Ce. represents the constant environment, and St. is the signal strength.

Table 1. Parameters used in implementation

\begin{tabular}{cc}
\hline Constraint & Rate \\
\hline Zone & $1500 \times 750$ \\
Node & 9 \\
Node Speed & $100 \mathrm{~m} / \mathrm{s}$ \\
Packet size & $32 \mathrm{byte}$ \\
Traffic Source & 9 \\
Constant environment & 2 \\
\hline
\end{tabular}

Table 2. Distance among nodes

\begin{tabular}{cccccccccc}
\hline & $\mathrm{A}$ & $\mathrm{B}$ & $\mathrm{C}$ & $\mathrm{D}$ & $\mathrm{E}$ & $\mathrm{F}$ & $\mathrm{G}$ & $\mathrm{H}$ & $\mathrm{I}$ \\
\hline $\mathrm{A}$ & 0 & 76 & 66 & 75 & 63 & 70 & 65 & 68 & 130 \\
$\mathrm{~B}$ & 76 & 0 & 10 & 1 & 13 & 6 & 11 & 8 & 4 \\
$\mathrm{C}$ & 66 & 10 & 0 & 9 & 3 & 4 & 1 & 2 & 4 \\
$\mathrm{D}$ & 75 & 1 & 9 & 0 & 12 & 5 & 10 & 7 & 5 \\
$\mathrm{E}$ & 63 & 13 & 3 & 12 & 0 & 7 & 2 & 5 & 17 \\
$\mathrm{~F}$ & 70 & 6 & 4 & 5 & 7 & 0 & 5 & 2 & 10 \\
$\mathrm{G}$ & 65 & 11 & 1 & 10 & 2 & 5 & 0 & 3 & 15 \\
$\mathrm{H}$ & 68 & 8 & 2 & 7 & 5 & 2 & 3 & 0 & 14 \\
$\mathrm{I}$ & 130 & 4 & 14 & 5 & 17 & 10 & 15 & 14 & 0 \\
\hline
\end{tabular}

Table 3. Power initiation per node

\begin{tabular}{lccccccccc}
\hline Node & A & B & C & D & E & F & G & H & I \\
\hline Power & 460 & 510 & 370 & 670 & 620 & 480 & 462 & 580 & 700 \\
\hline
\end{tabular}

The signal strength is calculated for each path in Table 4, which are used to calculate the best route with direct link as shown in Figures 4-6 respectively. I is parts of the following link and interference calculation to use formula 2 as shown below from node to node:

$$
S I N R=P t . * G(L L) / \mu+\sum P_{-} t^{\wedge^{\prime}} * G(L L) \geq \beta(r)
$$

The symbols of the peering node to node in the direct communication; where $\mathrm{L}$ is physical link, $\mu$ is receiver noise, $\mathrm{R}$ is transmission rate, $\mathrm{LL}$ is logical link between the origin to destination and $\in$ controllable for routing. Also, $\mathrm{G}(\mathrm{L})$ is path loss, $\beta(\mathrm{r})$ is bit error rate, power received at $\mathrm{d}(\mathrm{l})$ and proportional to $\mathrm{Pt}$. is $\mathrm{G}(\mathrm{L})$ path loss and $\mathrm{E}$ is the set of routing of the logical links. 
Table 4. Single strength among nodes

\begin{tabular}{cccccccccc}
\hline & $\mathrm{A}$ & $\mathrm{B}$ & $\mathrm{C}$ & $\mathrm{D}$ & $\mathrm{E}$ & $\mathrm{F}$ & $\mathrm{G}$ & $\mathrm{H}$ & $\mathrm{I}$ \\
\hline $\mathrm{A}$ & $\infty$ & 0.0796 & 0.1056 & 0.0818 & 0.1159 & 0.0939 & 0.1089 & 0.0995 & 0.0272 \\
$\mathrm{~B}$ & 0.0883 & $\infty$ & 5.1 & 510 & 3.0178 & 14.167 & 4.2149 & 7.9688 & 31.875 \\
$\mathrm{C}$ & 0.0849 & 3.7 & $\infty$ & 4.5679 & 41.111 & 23.125 & 370 & 92.5 & 1.8878 \\
$\mathrm{D}$ & 0.1191 & 670 & 8.2716 & $\infty$ & 4.6528 & 26.8 & 6.7 & 13.674 & 26.8 \\
$\mathrm{E}$ & 0.1512 & 3.5503 & 66.6667 & 4.1667 & $\infty$ & 12.2449 & 150 & 24 & 2.0761 \\
$\mathrm{~F}$ & 0.098 & 13.333 & 30 & 19.2 & 9.7959 & $\infty$ & 19.2 & 120 & 4.8 \\
$\mathrm{G}$ & 0.1093 & 3.8182 & 462 & 4.62 & 115.5 & 18.48 & $\infty$ & 51.333 & 2.0533 \\
$\mathrm{H}$ & 0.1254 & 9.0625 & 145 & 11.8367 & 23.2 & 145 & 64.4444 & $\infty$ & 2.9592 \\
$\mathrm{I}$ & 0.041 & 43.75 & 3.571 & 28 & 2.422 & 7 & 3.111 & 3.571 & $\infty$ \\
\hline
\end{tabular}

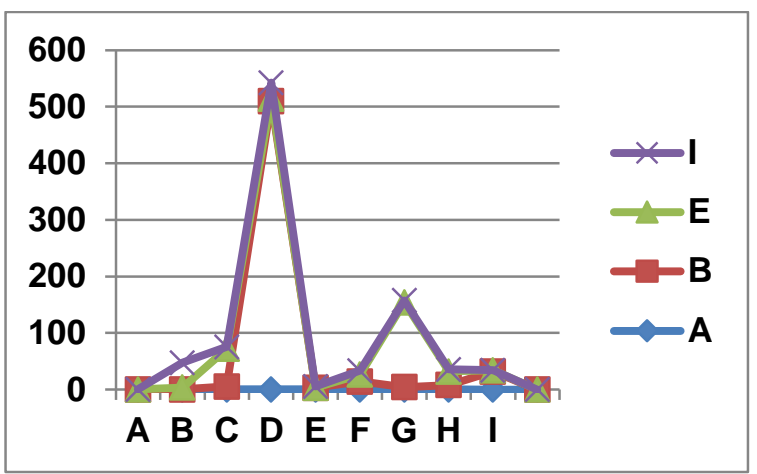

Figure 4. Route signal for path A, B, E, and I

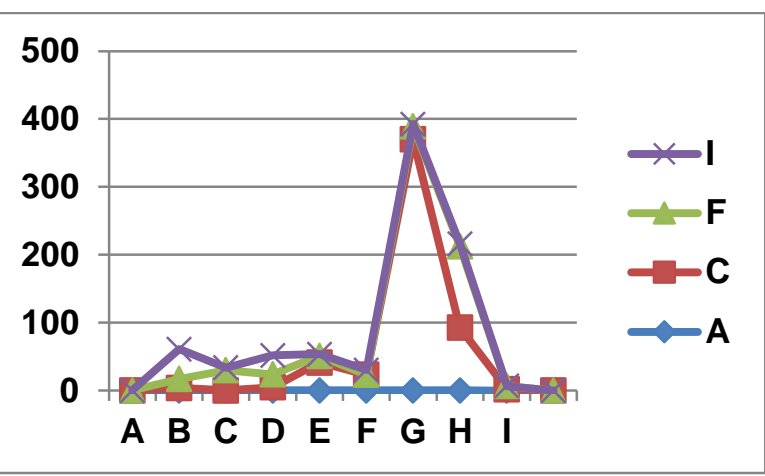

Figure 5. Route signal path A, C, F, and I

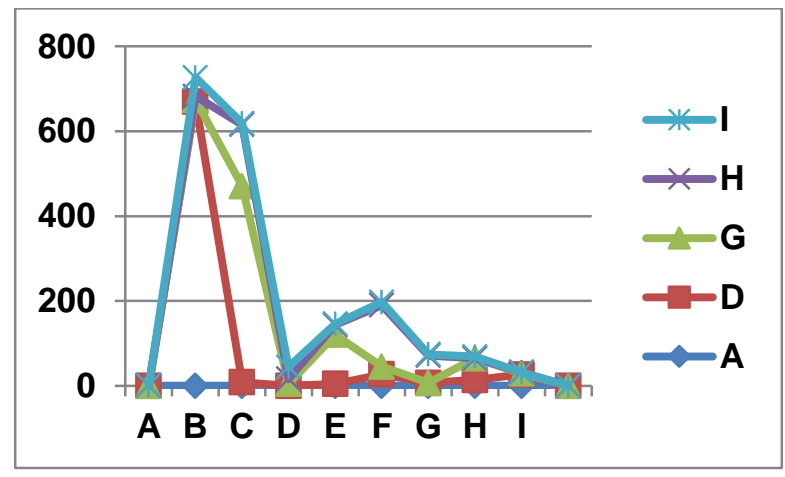

Figure 6. Route signal path A, D, G, H and I

\section{CONCLUSION}

The battery power for WMNs node is considered the main important issues in communication system especially for long distance. The propose solution in this paper is for the transmission data from node to node is designed to find an optimal data transmission path by using intermediate node or other nodes to send the packets form source to destination based on efficient distance between nodes. The environmental condition and the signal strength acquired when the distance between each node is less than the environment constant. This paper can be extended for future work to include other parameters such as transmission time, data size, etc.

\section{REFERENCES}

[1] P. Sharnya, and J. S. Raj, "Self Organizing Wireless Mesh Network," International Journal of Innovation and Applied Studies, vol. 3, no. 2, pp. 486-492, 2013.

[2] F. Zhang, et al., "Power Saving Access Points for IEEE 802.11 Wireless Network Infrastructure," 2004 IEEE Wireless Communications and Networking Conference (IEEE Cat. No.04TH8733), Atlanta, GA, USA, vol. 1, 2004, pp. 195-200. 
[3] IEEE Stds. Dept., "Part 11: Wireless Medium Access Control (MAC) and Physical Layer (PHY) Specifications: Medium Access Control (MAC) Quality of Service (QoS) Enhancements," IEEE Press, 2005.

[4] IEEE Stds. Dept., "Part 11: Wireless LAN Medium Access Control (MAC) and Physical Layer (PHY) specifications: Amendment 4: Enhancements for Higher Throughput," IEEE P802.11n/D3.00.IEEE Press, 2007.

[5] Z. Zeng, Y. Gao, and P. R. Kumar, "SOFA: A Sleep-Optimal Fair-Attention scheduler for the Power-Saving Mode of WLANs," 2011 31st International Conference on Distributed Computing Systems, Minneapolis, MN, 2011, pp. 87-98.

[6] J. Restrepo, C. Gruber, and C. Machuca, "Energy profile aware routing," in 2009 IEEE International Conference on Communications Workshops, Dresden, 2009., pp. 1-5.

[7] A. Oliva, A. Banchs and P. Serrano, "Throughput and energy-aware routing for 802.11 based mesh networks," Computer Communications, vol. 35, no. 12, pp. 1433-1446, 2012.

[8] Amokrane Ahmed, Langar Rami, Boutaba Raouf and Pujolle Guy, "A Green Framework for Energy Efficient Management in TDMA-based Wireless Mesh Networks," 2012 8th international conference on network and service management (cnsm) and 2012 workshop on systems virtualiztion management (svm), Las Vegas, NV, 2012, pp. 322-328.

[9] C. Bemmoussat, F. Didi, M. Feham, "Efficient Routing Protocol to support QOS IN Wireless Mesh Network," International Journal of Wireless \& Mobile Networks (IJWMN), vol. 4, no. 5, pp. 89-104, 2012.

[10] F. O. Aron, T. O. Olwal, A. Kurien, M. O. Odhiambo, "A Distributed Topology Control Algorithm to Conserve Energy in Heterogeneous Wireless Mesh Networks," Proceedings of World Academy of Science, Engineering and Technology, 2008, pp. 530-536.

[11] Praveen Gupta, Preeti Saxena, A. K. Ramani, Rajkamal Mittal, "Optimized Use of Battery Power in Wireless Ad hoc Networks," in 12th international conference on advanced communication technology, 2010.

[12] Ancillotti, Emilio, Carlo V., Raffaele B., and Enzo M., "A reinforcement learning-based link quality estimation strategy for RPL and its impact on topology management," Computer Communications, vol. 112, pp. 1-13, 2017.

[13] Q. Fan, J. Fan, J. Li, and Xiaofang Wang, "A Multi-hop Energy-efficient Sleeping MAC Protocol based on TDMA Scheduling for Wireless Mesh Sensor Networks," Journal of Networks, vol. 7, no. 9, 2012.

[14] B. Sansò S. Boiardi, A. Capone, "Energy Aware Planning and Management of Wireless Mesh Networks," 2012 IEEE Global Communications Conference (GLOBECOM), Anaheim, CA, 2012, pp. 3049-3055.

[15] Mothku, Sai Krishna, and Rashmi Ranjan Rout, "Adaptive fuzzy-based energy and delay-aware routing protocol for a heterogeneous sensor network," Journal of Computer Networks and Communications, 2019.

[16] A. Capone, F. Malandra, B. Sansò, "Energy Savings in Wireless Mesh Networks in a Time-Variable Context," Mobile Networks and Applications, vol. 17, no. 2, pp. 298-311, 2012.

[17] S. Mamechaoui, F. Didi, G. Pujolle, "A Survey on Energy Efficiency for Wireless Mesh Network," International Journal of Computer Networks \& Communications (IJCNC), vol. 5, no. 2, pp. 105-124, 2013.

[18] Amokrane A., Langar R., Boutaba R., Pujolle G. B., "Online flow-based energy efficient management in wireless mesh networks," 2013 IEEE Global Communications Conference (GLOBECOM), Atlanta, GA, pp. 329-335, 2013.

[19] Aguilar, S., Vidal, R., Gomez, C., "Opportunistic Sensor Data Collection with Bluetooth Low Energy," Sensors, vol. 17, no. 1, 2017, pp. 1-29.

[20] H. S. Kim, J. Lee, and J. W. Jang, "Blemesh: A wireless mesh network protocol for Bluetooth low energy devices," in 2015 3rd International Conference on Future Internet of Things and Cloud, Rome, 2015, pp. 558-563.

[21] L. Leonardi, G. Patti, and L. L. Bello, "Multi-hop real-time communications over bluetooth low energy industrial wireless mesh networks," IEEE Access, vol. 6, pp. 26505-26519, 2018.

[22] Anis Ouni, Hervé Rivano, Fabrice Valois, Catherine Rosenberg, "Energy and Throughput Optimization of Wireless Mesh Network with Continuous Power Control," IEEE Transactionson Wireless Communications, vol. 14, no. 2, pp. 1131-1142, 2015.

[23] Liang-Bi Chen, Bo-Chuan Cheng, You-Chiun Wang, Katherine Shu-Min Li, Jing-Jou Tang, "An efficient fault tolerance path finding algorithm for improving the robustness of multichannel wireless mesh networks," 2016 IEEE International Conference on Consumer Electronics (ICCE), Las Vegas, NV, 2016, pp. 524-525.

[24] Avallone S. and t Banchs A., "A Channel Assignment and Routing Algorithm for Energy Harvesting Multi-radio Wireless Mesh Networks," IEEE Journal on Selected Areas in Communications, vol. 34, no. 5, pp. 1463-1476, 2016.

[25] S. P. Shiva Prakash, T. N. Nagabhushan, and Kirill Krinkin, "On improving energy saving in IEEE 802.11s wireless mesh networks under mobility conditions," 2016 Second International Conference on Cognitive Computing and Information Processing (CCIP), Mysore, 2016, pp. 1-6.

\section{BIOGRAPHIES OF AUTHORS}

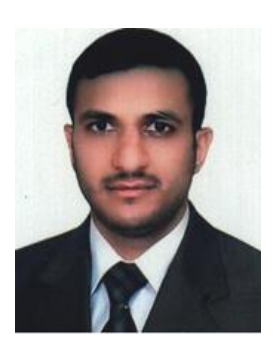

Mohammad Khalaf Rahim Al-juaifari Master of technology in computer science and engineering from JNTUH, Hyderabad 2012, India. Iam researcher and lecturer in University of Kufa, research interests are: wireless network power enhancement, virtualization, Data mining, I published 7 research papers and currently submit three under assignment, also I teached many topics in university of kufa such as Compiler Design, Digital System Design, Microprocessor. 

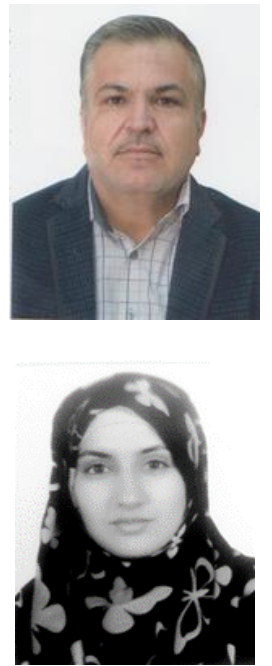

Hussain Mumtaiz Alshamy Lecturer at Kufa University/Engineering Science/Electronic and Communication department Demonstrated skills in solving technical problems and production issues - Coordinate production activates ensuring revenue and headcount targets are achieved and identified opportunities for continuous improvement activities •Led Health and Safety initiatives and ensured individuals take personal responsibility for providing a health and safe workplace. Extensive experience in the diagnoses and repair of a range of technologies at component level. Assisted in the development and management of projects, manufacturing changes, revenue targets and quality initiatives

Noor Hassan Abed Khammas She received B.Sc. degree in Electrical Engineering from University of Kuf /Iraq in 2009, and M.Sc. degree in Communications and Signal Processing Engineering from Newcastle University/UK in 2013. She joined the faculty of Engineering in University of Kufa since 2015. She is currently assistance lecturer at Electronics and Communications Engineering Department in University of Kufa. Her research interests focus on mobile and wireless communications, OFDM, OFDMA and she is interested also in wireless sensor networking. 